\title{
Uricase-deficient rat is generated with CRISPR/Cas9 technique
}

\author{
Yun Yu Equal first author, 1 , Nan Zhang Equal first author, ${ }^{1}$, Xianxiang Dong ${ }^{1}$, Nan Fan ${ }^{1}$, Lei Wang ${ }^{1}$, Yuhui Xu ${ }^{1}$, Huan Chen ${ }^{2}$, \\ Weigang Duan ${ }^{\text {Corresp. 1, } 2}$ \\ ${ }^{1}$ The Department of Pharmacology, School of Basic Medicine, Kunming Medical University, Kunming, Yunnan, China \\ 2 Yunnan Provincial Key Laboratory of Molecular Biology for Sinomedicine, School of Basic Medicine, Yunnan University of Traditional Chinese Medicine, \\ Kunming, Yunnan, China \\ Corresponding Author: Weigang Duan \\ Email address: duanweigang@ynutcm.edu.cn
}

Urate oxidase (uricase, Uox) is a big obstacle for scientists to establish stable animal models for studying hyperuricemia and associated disorders. Due to the low survival rate of uricase-deficient mice, we generated a Uox-knockout model animal from Sprague Dawley (SD) rats using the CRISPR/Cas9 technique by deleting exons 2 to 4 of the Uox gene. The uricase-deficient rats were named "Kunming-DY rats", and were apparently healthy with more than a $95 \%$ survival up to one year. The male rats' serum uric acid (SUA) increased to $48.3 \pm 19.1 \mu \mathrm{g} / \mathrm{ml}$, significantly higher than those of wild-type rats. Some indexes of the blood fat like total triglyceride, low density lipoprotein, and renal function indexes including blood urea nitrogen and serum creatinine were significantly different from those of wild-type rats, however, all the indexes were close to or in normal ranges. Histological renal changes including mild glomerular / tubular lesions were observed in these uricase-deficient rats. Thus, "Kunming-DY rats" with stable uricasedeficiency were successfully established and are an alternative model animal to study hyperuricemia and associated diseases mimicking human conditions. 


\section{Uricase-deficient rat is generated with CRISPR/Cas9}

\section{2 technique}

3 Yu Yun ${ }^{1 \#}$, Nan Zhang ${ }^{1 \#}$, Xianxiang Dong" ${ }^{1 \#}$, Nan Fan ${ }^{1}$, Lei Wang ${ }^{1}$, Yuhui Xu${ }^{1}$, Huan Chen ${ }^{2}$, Weigang

$4 \operatorname{Duan}^{1,2^{*}}$

51 The Department of Pharmacology, School of Basic Medicine, Kunming Medical University, Kunming

6 650500, China

72 Yunnan Provincial Key laboratory of Molecular Biology for Sinomedicine, School of Basic Medicine,

8 Yunnan University of Traditional Chinese Medicine, Kunming 650500, China

9

$10 \#$, contributed equally to this work.

11 Yu Yun: yunyu_li@126.com ; ORCID: 0000-0002-0030-2194

12 Nan Zhang: 1016117739@qq.com ; ORCID: 0000-0002-1825-8929

13 Xianxiang Dong: 1737346950@qq.com ; ORCID: 0000-0003-2554-5704

14 Nan Fan: 3324507727@.qq.com;

15 Lei Wang: 7176@163.com

16 Yuhui Xu: 562431311@qq.com

17 Huan Chen: qingxing1214@,163.com

18

19 Corresponding author:

20 Corresponding author: Weigang Duan*

21 Mailing address: Yunnan University of Traditional Chinese Medicine, 1076, Yuhua Rd.,

22 Kunming 650500, China

23 Tel.: +86-86-65919552; Fax: +86-86-65918014

24 Mobile phone: 18687106921

25 E-mail: deardwg@126.com ; ORCID: 0000-0002-9006-861X 


\section{Abstract}

Urate oxidase (uricase, Uox) is a big obstacle for scientists to establish stable animal models for studying hyperuricemia and associated disorders. Due to the low survival rate of uricase-deficient mice, we generated a

Uox-knockout model animal from Sprague Dawley (SD) rats using the CRISPR/Cas9 technique by deleting exons 2 to 4 of the Uox gene. The uricase-deficient rats were named "Kunming-DY rats", and were apparently healthy with more than a $95 \%$ survival up to one year. The male rats' serum uric acid (SUA) increased to 48.3 triglyceride, low density lipoprotein, and renal function indexes including blood urea nitrogen and serum conditions. animal; renal function; lipid metabolism 


\section{Introduction}

In recent decades, hyperuricemia and associated diseases have become common in modern society. Hyperuricemia is a well-known disorder of purine metabolism, and the disorder can be diagnosed by having a serum uric acid (SUA) above $70 \mu \mathrm{g} / \mathrm{ml}$ in men or above $60 \mu \mathrm{g} / \mathrm{ml}$ in women (Engel et al. 2017; Schlesinger 2017). The increase of SUA is caused either by uric acid over-synthesis, or by its insufficient excretion. Uric acid, as an end product from purines, is directly catalyzed by xanthine dehydrogenase (Xdh) in uricase-(urate oxidase, Uox) deficient creatures including humans. Uric acid and its salts are polar compounds but with poor solubility (Iwata et al. 1989). The redundant uric acid and its salts are precipitated in the kidneys, bones and joints as tophi, usually resulting in renal dysfunction and gout (Feig 2014); however, the relationship between hyperuricemia and other diseases is still unclear (Borghi et al. 2019).

Uric acid can be further transformed by uricase and other enzymes to more soluble chemicals (Ramazzina et al. 2006). The transformation prevents uric acid from precipitation in body fluids. However, Uox is a pseudo-gene in humans, and other mammals like apes, elephants and manatees (Sharma \& Hiller 2019), and the gene in them cannot be translated to uricase (Keebaugh \& Thomas 2010). Therefore, the deficiency of uricase makes humans vulnerable to hyperuricemia and relevant diseases though it is believed to be important for human evolution (Alvarez-Lario \& Macarron-Vicente 2010).

As for researching hyperuricemia and for evaluating uric-acid-lowering drugs, naturally uricase-deficient mammals could be ideal experimental animals. However, they are not commonly used in laboratories by scientists. Instead, scientists use rats or mice for the convenience of breeding. Since rats and mice express uricase, a uricase inhibitor, potassium oxonate was usually applied to mimic human's purine metabolism (Mangoff \& Milner 1978; Stavric \& Nera 1978). Nevertheless, the extra inhibitor has multiple activities, which would make the animal model tangled with multiple factors (Stavric \& Nera 1978). To overcome the shortcomings mentioned above, scientists knocked out or modified mice's Uox gene by homologous recombination in embryonic stem cells (Wu et al. 1994), or by using the transcription activator-like effector nuclease (TALEN) technique (Lu et al. 2018) to successfully breed heterozygote mice. However, it is still difficult for scientists to breed apparently healthy homozygote animals to mimic the human condition. The homozygote mice would die by 11 weeks after birth because of renal failure (Wu et al. 1994), or displayed a 
low survival rate even kept in sterile environments ( $\mathrm{Lu}$ et al. 2018). These findings suggested that it is a big challenge to breed artificial uricase-deficient $\left(\mathrm{Uox}^{-/-}\right)$animals. Considering that most naturally uricase-deficient animals are healthy, or at least apparently healthy, the biological features of the uricase-deficient mice could be deviated from humans to some extent.

Consequently, the knock-out or gene-modified mice left researchers with a limited choice for a model animal to study hyperuricemia. However, rat is another common experimental animal, whose Uox gene has not knocked out or modified. Here, to establish an alternative model animal, we tried to generate healthy uricasedeficient rats, called "Kunming-DY rats", with the CRISPR/Cas9 technique.

\section{Materials and methods}

\section{Materials}

Wild-type Sprague Dawley (SD) rats were obtained from Jianyang Dashuo Science and Technology Ltd., Chengdu, China [Certification No. SCXK (Chuan) 2008-24]. Rats were maintained at $22{ }^{\circ} \mathrm{C}$, with a humidity of $45 \%-55 \%$ under natural light and with free approach to food and water. All animal experiments were approved by Animal Care and Use Committee of Yunnan Provicial Key Laboratory of Molecular Biology for Sinomedicine (Approved No. LL-20170930-01), Yunnan University of Traditional Chinese Medicine. All the animal experiments were performed in accordance with Guidelines for Ethical Review of Laboratory Animal Welfare of China.

Uric acid was purchased from Tokyo Into Industrial Co., Ltd. (Tokyo, Japan). Standard solution of uric acid $(1000 \mu \mathrm{g} / \mathrm{ml}, 5952 \mu \mathrm{mol} / \mathrm{l})$, uric acid assay kits of phosphotungstic acid method (Lot: C012-1-1), blood urea nitrogen (BUN) assay kits of diacetyl monoxime method (Lot: C013-1-1), serum creatinine (Cr) of sarcosine oxidase method (Lot: C011-2-1), and protein assay kits of BCA (bicinchoninic acid) method (Lot: A045-4-2) were purchased from Nanjing Jiancheng Bioengineering Institute (Nanjing, China). Total triglyceride (TG) assay kits of glycerol lipase oxidase (GPO-PAP) method (Lot: A110-1-1), total cholesterol (TC) assay kits of GPO-PAP method (Lot: A111-1-1), low-density lipoprotein (LDL) assay kits (Lot: A113-11), high-density lipoprotein (HDL) assay kits (Lot: A112-1-1), and serum glucose kits of glucose oxidase 
124

method (Lot: F006-1-1) were also purchased from Nanjing Jiancheng Bioengineering Institute (Nanjing, China). TRIzol Plus RNA Purification kit was purchased from Introgen (Carlsbad, CA, USA). Rabbit antimouse Uox (Lot: bs-6716R) and mouse anti- $\beta$-actin (Lot: bsm-33036M) were purchased from Beijing Bioss Antibodies (Beijing, China). Goat anti- rabbit antibody linked with HRP (Lot: BA1055) and goat anti-mouse antibody linked with HRP (Lot: BA1051) were purchased from Boster Biological Engineering Co., Ltd (Wuhan, China). Enhanced chemoluminescence (ECL) detection kits (Lot: 34059) were purchased from Pierce Biotechnology Inc (Rockford, IL, USA).

Ultra-pure water was produced with a Milli Q water purification system manufactured by EMD Millipore Group (Darmstadt, Germany). The NanoDrop ND-1000 spectrophotometer used for experiments was manufactured by PeqLab (Erlangen, Germany). The multimicroplate reader of Infinite 200pro used was manufactured by Tecan Group (Mannedorf, Switzerland). A fluorescence microscope was manufactured by Olympus Corp. (Tokyo, Japan). Other instruments or reagents used in the present study were made in China if not mentioned.

\section{Design and generation of $\mathrm{Uox}^{+/-}$rat}

The CRISPR/Cas9 technical design and operation of generation of $\mathrm{Uox}^{+/-} \mathrm{SD}$ rat were pre-formed by Cyagen Bioscience Inc. (www.cyagen.com). Briefly, the Uox gene is located on chromosome 2 (NM_053768.2; Ensembl: ENSRNOG00000016339) with 8 exons. Eight exons have been identified, with the ATG start codon in Exon 1 and TGA stop codon in Exon 8. Three exons (Exon 2 to Exon 4) were selected as target sites. The two pairs of gRNA targeting vectors were constructed and confirmed by sequencing. Cas9 mRNA and gRNA generated by in vitro transcription were co-injected into fertilized eggs for deficient rat production. The pups were genotyped by PCR followed by sequence analysis. The gRNA1 (matches forward strand of gene) was CCACTAGGCTAGGCGTAGCA $\underline{A G G}$, and gRNA2 (matches reverse strand of gene) was TTTTCATATTGACTGACGGCAGG. The links of gRNA on VectorBuilder can be found at https://www.vectorbuilder.com/vector/VB171130-1198hbq.html and https://www.vectorbuilder.com/vector/VB171130-1199dvz.html (gRNA2). The target region of rat Uox locus was amplified by PCR with specific primers. PCR product was sequenced to confirm targeting. The primer sequence was 
125

126

127

128

129

130

131

132

133

136

137

138

GAGGTCTGTGCGTCTCTCCTCATC (reverse). The PCR product was expected to be 6445 bp in wild-type allele and $\sim 600$ bp in mutant allele, suggesting $\sim 6840$ bp would be deleted.

\section{Generation of $\mathbf{U o x}^{-/-}$rat}

Uox $^{+/-}$rats were generated and identified by Cyagen Bioscience Inc. They were housed in a SPF (specific pathogen free) environment, maintained at $22^{\circ} \mathrm{C}$ temperature, at $45 \%-55 \%$ humidity-controlled conditions, and under a 12-hour light-dark cycle with free access to irradiated rodent diet and sterile water. The male and female animals of heterozygote $\left(\mathrm{Uox}^{+/-}\right)$were matched to generate $\mathrm{Uox}^{-/-}$offspring. Likely Uox ${ }^{-/-}$offspring were screened by their SUA level at the first round. The likely Uox ${ }^{-/}$male and female rats from the same parental rats were also mated to generate stable $\mathrm{Uox}^{-/-}$offspring. When their offspring grew up, the parental likely $\mathrm{Uox}^{-/}$rats were further identified by their enzyme activity, protein expression, and mRNA expression. The phenotype and molecular biological identification were taken until stable $\mathrm{Uox}^{-/-}$rats were obtained. The $\mathrm{Uox}^{-/-}$rats of the second to the sixth generation (F2 to F6) were used to investigate their biological features including body weight, SUA, BUN, Cr, and serum glucose.

\section{Blood and organ sampling}

Rats were kept in a small cage, and their blood sample of $0.5 \mathrm{ml}$ was drawn from their tail with a tiny needle without anesthetization at a local atmosphere of $28^{\circ} \mathrm{C}-32^{\circ} \mathrm{C}$. Serum was obtained by spinning at $3,000 \times \mathrm{g}$ and at $4^{\circ} \mathrm{C}$ for $5 \mathrm{~min}$. Serum was used for valuating uricase activity, SUA, BUN, Cr, blood fat or serum glucose.

In order to obtain tissue samples, rats were intraperitoneally anaesthetized with urethane $(1.0 \mathrm{~g} / \mathrm{kg})$. Their abdomens were opened, and their livers and kidneys were harvested. Their livers were homogenated to extract mRNA for RT-PCR and evaluate tissue uricase activity. Their left kidneys were used for evaluating the left kidney index and used for RNA extraction, and their right kidneys were used for histological examination. The left kidney was weighted and the left kidney index (LKI) was calculated by Formula-1.

Left Kidney Index $(L K I)=\frac{\text { weight }_{\text {left kidney }}}{\text { weight }_{\text {body }}}($ Formula-1)

Except rats for breeding, other rats at the conclusion of the experiment were intraperitoneally anesthetized 
150

151

152

153

154

with urethane $(1.0 \mathrm{~g} / \mathrm{kg})$, and their necks were dislocated for euthanasia.

\section{Uricase activity assay}

The maximum absorption wavelength of uric acid in weakly alkaline solution is $292 \mathrm{~nm}$, and the absorbance at the wavelength (A292) can be used to evaluate the level of uric acid in the solution. Enough uric acid powder was dissolved in $50 \mathrm{mmol} / 1 \mathrm{NaHCO}_{3}$ solution to make a uric acid saturated solution (about 1500 $\mu \mathrm{g} / \mathrm{ml}, 20^{\circ} \mathrm{C}$ ), and the supernatant of the solution was transferred to another tube and diluted with $50 \mathrm{mmol} / \mathrm{l}$ $\mathrm{NaHCO}_{3}$ to adjust its A292 (Ma et al. 2018) of a $95 \mu$ l solution in a 96-well plate from 1.5 to 2.0 (namely, 95 $\mu \mathrm{l}$ uric acid solution of $45-50 \mu \mathrm{g} / \mathrm{ml})$. Five microliters of serum was added to $95 \mu \mathrm{l}$ of uric acid solution and mixed at $37{ }^{\circ} \mathrm{C}$. An A292 of the mixture was read at an interval of 5 min for $240 \mathrm{~min}$. By subtracting the A292 value caused by serum and the naturally degraded uric acid in a $50 \mathrm{mmol} / 1 \mathrm{NaHCO}_{3}$ solution, the net $\mathrm{A} 292$ decrease $(\triangle \mathrm{A} 292)$ was used to evaluate uricase activity. Serum of wild-type rats contains uricase, Xdh, xanthine and uric acid. At the beginning of the reaction, A292 was transiently increased in the reaction system containing wild-type rat serum. The increase of A292 may have resulted from uric acid synthesis caused by Xdh.

Similarly, liver tissue from rats was homogenated on ice, and the supernatant was obtained by spinning at $3,000 \times \mathrm{g}$ and at $4^{\circ} \mathrm{C}$ for $5 \mathrm{~min}$. The supernatant was diluted, and the uricase activity was assayed in the same reaction system as mentioned above.

\section{Western blot}

Rat liver tissue (about $100 \mathrm{mg}$ ) was homogenated in icy isotonic lysis buffer ( $25 \mathrm{mmol} / \mathrm{l}$ Tris, $\mathrm{pH} 7.4,150$ $\mathrm{mmol} / 1 \mathrm{NaCl}$, complete protease inhibitors from Roche (Complete, EDTA-free, glass vials, Lot: 11873580001, $1 \mathrm{mmol} / 1$ sodium orthovanadate, $1 \mathrm{mmol} / 1$ sodium pyrophosphate, $10 \mathrm{mmol} / 1 \beta$-glycerophosphate). The protein supernatant for Western blot was obtained by spinning the homogenate at $5,000 \times \mathrm{g}$ and at $4^{\circ} \mathrm{C}$ for $5 \mathrm{~min}$. Protein in the supernatant was determined by the protein assay kit of BAC method. The protocol of Western blot was described previously (Yun et al. 2017). Briefly, protein $(50.0 \mu \mathrm{g}$ ) was applied on to $10 \%$ sodium dodecyl sulfate-polyacrylamide gel electrophoresis (SDS-PAGE) and the protein was separated by a direct current at $100 \mathrm{~V}$. The protein in the SDS-PAGE was transferred to a nitrocellulose (NC) membrane by a direct 
176

177

178

179

180

181

182

183

184

185

186

187

188

189

190

191

192

193

194

195

196

197

198

199

200

201

202

current at $10 \mathrm{~V}$ for $60 \mathrm{~min}$ in a semi-dry electrophoretic transfer cell. The NC membrane was blocked with 3\% bovine serum albumin at an ambient temperature for $2 \mathrm{hr}$ and bathed in the primary antibody solution (1:400) at $4^{\circ} \mathrm{C}$ overnight. The membrane was rinsed with TST Buffer $(20 \mathrm{mmol} / 1$ Tris- $\mathrm{HCl}, \mathrm{pH} 7.5,0.05 \%$ Tween-20) for 10 min three times, and bathed in a solution of goat anti-rabbit antibody linked with HRP $(1: 800)$ for Uox detection or in a solution of goat anti-mouse antibody linked with HRP (1:800) for $\beta$-actin detection for another $2 \mathrm{hr}$. The NC membrane was rinsed with TST buffer for 10 min three times and developed by the ECL detection kits. The band brightness was quantified with ImageJ 1.48v software. Uricase protein from wild-type rat is about $35 \mathrm{kDa}$, and $\beta$-actin protein is about $42 \mathrm{kDa}$.

\section{RT-PCR and high throughput sequencing}

Rat liver and kidney (about $100 \mathrm{mg}$ ) were frozen with liquid nitrogen and ground to powder. The total RNA in the powder was extracted and purified with a TRIzol Plus RNA Purification kit. The RNA quantity and quality were measured by a NanoDrop ND-1000 spectrophotometer. RNA integrity was assessed by standard denaturing agarose gel electrophoresis (denatured by $0.8 \%$ methanal), and three bands in one lane suggested its integrity (Chen et al. 2017; Yin et al. 2015).

Double-stranded cDNA (ds-cDNA) from liver was synthesized from an aliquot of total RNA using an Invitrogen SuperScript ds-cDNA synthesis kit in the presence of $100 \mathrm{pmol} / 1$ oligo dT primers. The ds-cDNA of Uox was amplified with a pair of primers: 5 '-CCCATTACCATGACGACTATGG-3'(forward) and 5'GGACCTCCCATCATTCACTCTG-3'(reverse), and that of glyceraldehyde 3 phosphate dehydrogenase (GAPDH, NM_017008.4) was amplified with another pair of primers: 5`-TGTGAACGGATTTGGCCGTA$3^{\prime}$ (forward) and 5'-TGAACTTGCCGTGGGTAGAG-3`(reverse). Briefly, a template (ds-cDNA) of $1 \mu$, primers of 2 nmole and the Taq mixture (Sangon Biotech, Shanghai, China; Lot: B110006) were mixed together to $50 \mu$ l. The solution of $50 \mu \mathrm{l}$ was incubated in a PCR machine (Life Express Gradient, Hangzhou Bioer Technology Co., Ltd., Hangzhou, China) $94^{\circ} \mathrm{C}$ for $60 \mathrm{~s}, 55^{\circ} \mathrm{C}$ for $60 \mathrm{~s}, 72^{\circ} \mathrm{C}$ for $90 \mathrm{~s}, 30$ cycles, and finally kept at $72^{\circ} \mathrm{C}$ for $20 \mathrm{~min}$. The Uox product of PCR from wild-type rats is about $500 \mathrm{bp}$ (462 bp), and that from Uox ${ }^{-/}$rat should be much shorter or not exist. GAPDH was used as control, and its product of PCR is $149 \mathrm{bp}$.

Another aliquot of total RNA from liver was sequenced with the second generation of high throughput 
203

204

205

206

207

208

209

210

211

212

213

214

215

216

217

218

219

220

221

222

223

224

225

226

227

228

229

sequencing technique by Sangon Biotech (Shanghai, China). Fragments of $150 \mathrm{nt}$ from Uox mRNA in liver tissue were obtained by high throughput sequencing. The raw data in fastq (fq) format was transformed to original sequences in fasta (fa) format by Seqkit software in a DOS (disc operation system) model (Shen et al. 2016). Sequences matching 27 bp or more of Uox mRNA (NM_053768) were screened out by TBtools software (v0.664445552) in "Auto Blast Several Sequences To a Big File" model. By using the reference Uox mRNA as a template, matched sequences were used to restore the original mRNA of Uox ${ }^{-/}$rat (Conesa et al. 2016).

Total RNA from rat kidney was also sequenced with the second generation of high throughput sequencing technique by Sangon Biotech. Expected values of fragment per kilobases of exon model per million mapped reads (FPKM) were calculated from mapped reads, and were used for normalization of gene expression level (Lin et al. 2017; Trapnell et al. 2010). Values of FPKM were used to evaluate the level of gene expression associated with urate excretion or reclamation in kidney.

\section{SUA, BUN, Cr, blood fat and serum glucose determination}

Blood samples were drawn from tails as mentioned above. When the blood coagulated, serum was obtained by spinning at $1,000 \times \mathrm{g}$ for $10 \mathrm{~min}$. The SUA $(\mu \mathrm{g} / \mathrm{ml})$ was determined with uric acid assay kits according to the protocol provided by the producer. BUN (mmol/l) and $\mathrm{Cr}(\mathrm{nmol} / \mathrm{l})$ in the serum samples were determined with urea assay kits and creatinine assay kits according to the protocols provided by the producer, respectively. Blood fat indexes including TG, TC, LDL, and HDL in serum and serum glucose were determined with assay kits according to the protocols provided by the manufacturer. All the protocols can be seen or downloaded from websites: http://www.njjcbio.com/uploadfile/product/big/20190612093216738.pdf for uric acid assay; http://www.njjcbio.com/uploadfile/product/big/20190612093249433.pdf for BUN assay; http://www.njicbio.com/uploadfile/product/big/20190612093006383.pdf

for

for http://www.njicbio.com/uploadfile/product/big/20190611151410510.pdf

http://www.njicbio.com/uploadfile/product/big/20190611151511722.pdf

http://www.njicbio.com/uploadfile/product/big/20190611151926041.pdf http://www.njicbio.com/uploadfile/product/big/20190611151745050.pdf http://www.njjcbio.com/products.asp?id=812 for glucose assay. 


\section{Histological examination of kidney sections}

231

232

233

234

235

236

237

238

239

240

241

242

243

244

245

246

247

248

249

250

251

252

253

254

255

Rats' right kidneys were immersed in 4\% paraformaldehyde solution until a routine Hematoxylin-eosin (HE) staining was conducted. Paraffin-embedded sections $(5 \mu \mathrm{m})$ of the organs were cut. The sections were stained with an HE staining kit (Boster Biological Engineering Co., Ltd Wuhan, China). Images covered renal cortex or medulla were visualized with the fluorescence microscope in a light mode. The scoring systems used were defined as follows: glomerular abnormality: 0, normal glomeruli; 1, rare single abnormal glomerulum; 2 , several clusters of abnormal glomeruli; 3, massive abnormality; tubular atrophy: 0, normal tubules; 1 , rare single atrophic tubule; 2 , several clusters of atrophic tubules; 3 , massive atrophy; tubular necrosis: 0 , normal tubules; 1 , rare single necrotic tubule; 2 , several clusters of necrotic tubules; 3 , massive necrosis; lymphocytic infiltrates: 0 , absent; 1 , few scattered cells; 2, groups of lymphocytes; 3 , widespread infiltrate; interstitial fibrosis: 0 , absent; 1, minimal fibrosis, with slight thickening of the tubular basal membrane; 2, moderate fibrosis, with focal enlargement of the interstitium; 3, severe fibrosis, with confluent fibrotic areas (Debelle et al. 2002).

\section{Statistical analyses}

Values were expressed as mean $\pm \mathrm{SD}$ (standard deviation). If normal distribution of values was verified by normality test (Shapiro-Wilk test), independent Student's t test (two-tailed) was performed to compare means between groups. Otherwise, nonparametric test for two independent samples in Mann-Whitney U model (two tailed) was performed. Statistical significance was accepted at $\mathrm{P}<0.05$.

\section{Results}

\section{Generation of $\mathrm{Uox}^{+/-}$rat}

The CRISPR/Cas 9 experiment took four rounds to generate $\mathrm{Uox}^{+/}$rats. In the first two rounds, CRISPR/Cas9 operation was performed successfully, but no Uox $^{+/-}$rats were generated, possibly because of off-target. In the third and fourth rounds, CRISPR/Cas9 experiments were improved, and $6 \mathrm{Uox}^{+/-}$rats including three female and three male rats were generated. The $\mathrm{Uox}^{+/-}$rats were identified with gene sequencing by Cyagen Biosciences Inc. Uox $^{+/}$rats were apparently healthy, and no abnormal phenomenon 
256

257

258

259

260

261

262

263

264

265

266

267

268

269

270

271

272

273

274

275

276

277

278

279

280

281

was observed. When they were 2 months old, their SUA was determined (Table 1).

\section{First generation of $\mathrm{Uox}^{-/-}$rat}

When $\mathrm{Uox}^{+/-}$rats were 2 months old, a female and a male Uox ${ }^{+/-}$rats from the same round were matched and kept in one cage to generate Uox $^{-/}$offspring. About 3 weeks or more after they mated, their offspring were born. The likely Uox ${ }^{-/}$offspring were screened by the level of SUA 45 days after birth, or identified after their natural death (if happened). In our experimental system, the SUA in adult male rats of the heterozygote type is less than $20 \mu \mathrm{g} / \mathrm{ml}$ (Table 1), and not higher than $40 \mu \mathrm{g} / \mathrm{ml}$ (male) or $30 \mu \mathrm{g} / \mathrm{ml}$ (female). Therefore, male rats with an SUA above $40 \mu \mathrm{g} / \mathrm{ml}$ or female rats with SUA above $30 \mu \mathrm{g} / \mathrm{ml}$ were recognized as likely Uox $^{-/-}$rats. According to our previous study, uric acid in wild-type rats' kidney tissue is $174.51 \pm 19.61 \mu \mathrm{g} /$ (g tissue) (Yun et al. 2017). As for the dead offspring, if their uric acid levels in their kidney tissue were much higher than that in wild-type rats, they can also be recognized as likely Uox ${ }^{-/}$rats after autopsy. However, only one female rat's likely Uox $^{-/}$offspring were obviously healthy. The other two female rats' Uox ${ }^{-/}$offspring died several days after birth. Namely, offspring from the third round $\mathrm{Uox}^{+/-}$rats died out, and only offspring from the fourth round $\mathrm{Uox}^{+/-}$rats survived (Table 2). Autopsies of dead rats showed no obvious abnormal organ changes and particularly no visible calculus or sediments were found in kidneys and ureters. The likely Uox ${ }^{-/-}$ rats that survived were further identified with molecular biological techniques at enzyme, protein and mRNA levels after their offspring were generated. The identified Uox ${ }^{-/}$rats were named "Kunming-DY" rats.

\section{$\mathrm{Uox}^{-/-}$rats identification at enzyme level}

The increase in A292 was followed by a decreased caused by serum uricase from wild-type rat. As for the reaction system containing Uox $^{-/-}$rat serum, A292 continuously increased, suggesting there were no uricase activity in Uox $^{-/-}$rat serum (Fig. 1A). Similar increase was also obtained in liver homogenate from Uox $^{-/-}$rat (Fig. 1B), though there were differences between serum and liver homogenate in wild-type rat.

\section{Uox $^{-/-}$rats identification by Western blot and RT-PCR}

Peer) reviewing PDF | (2020:01:44904:2:0:NEW 19 Mar 2020) 

wild-type rat can do so.

\section{mRNA mapping}

The fragments were BLASTed with the mRNA sequence (NM_053768.2), and more than 6000 positive

reads were screened out. The results of mRNA mapping based on the positive reads were showed in Fig. 3 .

The horizontal axis was the reads, and vertical axis showed the sequence of the template mRNA

(NM_053768.2). A striped bar means a fragment of a read or the whole read mapped the template mRNA. If there were two or more reads that mapped the same fragment, two or more striped bars were showed behind. The Uox mRNA fragments mapped by reads of wild-type rat almost fully covered the template mRNA (36$1359 \mathrm{nt}$ ) (see the vertical axis, Fig. 3A). However, some part (1-35 nt and 71-477 nt) was missing in Uox ${ }^{-/-}$ rat's mRNA (see the vertical axis, Fig. 3B). After further comparison, it was found that, nucleotide segments from Exon 2, Exon 3 and Exon 4 were completely deleted, and the restored mRNA only contained 917 nt, much shorter than the original one (1359 nt). Therefore, it is impossible for the modified mRNA to translate the right uricase protein. identified by molecular biological techniques. To our surprise, their offspring of the second to the sixth generation were apparently healthy. The numbers of offspring from a couple of parental $\mathrm{Uox}^{-/-}$rats varied from

\section{Stable $\mathrm{Uox}^{-/-}$rats in general}

Adult $\mathrm{Uox}^{-/-}$rats of the first generation produced by parental $\mathrm{Uox}^{+/-}$rats were screened out by SUA and 6 to 15 , and the sex ratio is 0.865 (Male:female $=160: 185$ ). When all the offspring were identified as Uox $^{-/-}$ rats, it can be concluded that their offspring are stable for uricase-deficiency. The survival rate of the five generations (F2-F6) for two months was 99.4\% (343/345). The survival rate of the first two generations for one year is $95 \%$ in 20 rats (10 male and 10 female), because only one female died of unknown causes. There are no obvious abnormal behaviors without artificial intervention. At the same age, male rats were heavier than the female (Fig. 4A). However, comparing with wild-type SD rats of the same sex, the differences were not significant. 


\section{SUA, blood fat and serum glucose in stable Uox $^{-/-}$rats}

311 The SUA in wild-type rats and heterozygote type rats was under $20 \mu \mathrm{g} / \mathrm{ml}$ in the present study, though the 312 SUA in male rats of wild-type was higher than that in the female $(\mathrm{P}<0.05)$ (Fig 4B). However, the SUA in 313 Uox $^{-/-}$male and female rats (Fig. 4C) were elevated compared to that in wild-type rats $(\mathrm{P}<0.05)$ (See Fig. 4B). 314 Similarly, the SUA in male Uox $^{-/-}$rats $(48.4 \pm 19.1)$ was significantly higher than that in females $(39.9 \pm 20.8)$ 315 (Fig. 4C) $(\mathrm{P}<0.05)$.

316 Both in wild-type rats and in Uox ${ }^{-/-}$rats, the values of TG, TC, HDL, and LDL were close to or in normal 317 ranges (TG, 0.56-1.7 mmol/1; TC, 3.8-6.1 mmol/1; HDL, 0.78-2.2 mmol/1; and LDL, 2.07-3.1 mmol/l), though 318 the values of TG and LDL in $\mathrm{Uox}^{-/-}$rats were significantly different from those in wild-type rats (Fig. 4D).

319 Serum glucose in $\mathrm{Uox}^{-/-}$rats was $6.96 \pm 1.94 \mathrm{mmol} / \mathrm{l}(\mathrm{n}=10)$, also close to the normal range $(5.65 \pm 1.63$ $320 \mathrm{mmol} / \mathrm{l})$.

\section{Kidney and its function in $\mathbf{U o x}^{-/-}$rats}


335 injures occurred in Uox $^{-/}$rats. Their glomeruli were enlarged and their capsular spaces were slightly dilated

336 (Fig. 6C). The tubular walls became thin, and the tubular spaces were also slightly dilated (Fig. 6D). Sporadic 337 interstitial fibrosis and inflammatory cell infiltration can also be seen in Uox ${ }^{-/-}$rat kidneys (Fig. 6C and D). In 338 general, the renal histological changes was mild but with significance $(\mathrm{P}<0.05)$ (Table 4).

\section{Discussion}

Model animals highly mimicking human purine metabolism are necessary for biomedical scientists who study hyperuricemia and associated diseases and for pharmacologists who study uric acid-lowering drugs. Though there are uricase-deficient mice (Lu et al. 2018; Wu et al. 1994), uricase-deficient rats could be a better choice. CRISPR/Cas9 technique (Sapranauskas et al. 2011) is a recent favorite method used most frequently to obtain gene modified animals (Nakamura et al. 2019), but there are often reports of off-target effects (Li et al. 2019). Therefore, F0 animals and their offspring should be identified until their stable offspring present expected biological feature. Fortunately, after repeated hybridization and identification, we were able to generate a Uox ${ }^{-/-}$rat by deleting three exons (from Exon 2 to Exon 4) of the enzyme gene. Unlike Uox ${ }^{-/-}$mice (Lu et al. 2018; Wu et al. 1994), more than $95 \% \mathrm{Uox}^{-/-}$rats are apparently healthy and can survive for more than one year. Like wild-type rats, most parental $\mathrm{Uox}^{-/-}$rats can generate apparently healthy offspring. In honor of Kunming city and its founders, the Uox ${ }^{-/}$rat was given the name "Kunming-DY rat".

Unlike the SUA levels in Uox ${ }^{-/-}$mice, the SUA levels in $\mathrm{Uox}^{-/-}$rats were not as high as expected (Lu et al. 2018; Wu et al. 1994), although much higher than that in wild-type rats. Mice are small animals, while rats are relative larger than them. Usually, small animals have a much shorter life cycle, meaning that their fast cellular turnover, accompanied with higher metabolic rate, would cause the significant uric acid increase (Liu et al. 2019). According to the values tested, the SUA in male $\mathrm{Uox}^{-/-}$rats $(48.3 \pm 19.1 \mu \mathrm{g} / \mathrm{ml})$ was proportional to that of men $(25-70 \mu \mathrm{g} / \mathrm{ml})$. In the adult male Uox ${ }^{-/}$rats, only a small portion of rats' SUA was above $70 \mu \mathrm{g} / \mathrm{ml}$. The results suggested that the SUA in all $\mathrm{Uox}^{-/}$rats is not high enough for a hyperuricemia diagnosis. To establish a "real" hyperuricemia (SUA $>70 \mu \mathrm{g} / \mathrm{ml}$ ) model, other treatments, like increasing purine intake or limiting urate excretion, should be added.

Fortunately, obvious abnormally functional features, especially obvious disorders of blood fats and serum 
362

363

364

365

366

367

368

369

370

371

372

373

374

375

376

377

378

379

380

381

382

383

384

385

386

387

388

389

390

391

392

glucose were not observed in the Uox ${ }^{-/}$rats. Though deficiency of uricase can significantly affect TG and LDL levels, the values of all the indexes evaluating blood fat were close to or in the normal ranges. In addition, the values of BUN and $\mathrm{Cr}$, common indexes to evaluate renal function, were also close to or in the normal ranges, though BUN in $\mathrm{Uox}^{-/-}$rats was significantly higher than that in wild-type rats. However, this absence of abnormal function does not mean that uricase-deficiency has no negative impact on rats' renal function. Though the adult $\mathrm{Uox}^{-/-}$rats were apparently healthy, their compensatory renal function was impaired, as demonstrated in the histological examination, which showed slight glomerular and tubular lesion. Considering the rats were able to survive more than one year, the renal injury was much less than that in Uox ${ }^{-/-}$mice $(\mathrm{Lu}$ et al. 2018; Wu et al. 1994). Indeed, the present data suggested that the animal may be vulnerable to substances with renal toxicity, and even more sensitive to factors causing metabolic syndrome. Considering the low survival rate of $\mathrm{Uox}^{-/-}$mice (Lu et al. 2018; Wu et al. 1994) and the high survival rate of large uricase-deficient creatures like humans, elephants and manatees (Sharma \& Hiller 2019), uricase may be a key enzyme for small mammal's survival. In addition, compared with the level of SUA in Uox ${ }^{-/}$mice, the lower SUA in Uox $^{-/-}$rats could be associated with urate renal excretion, which needs further investigation.

\section{Conclusions}

Because Uox $^{-/-}$rats were uricase-deficient, their purine metabolism, especially their SUA features, is much similar to humans'. In conclusion, the $\mathrm{Uox}^{-/-}$rat is an alternative model animal for studying hyperuricemia and associated disorders, though it needs further investigation to evaluate the animal's other biological features.

\section{References}

Alvarez-Lario B, and Macarron-Vicente J. 2010. Uric acid and evolution. Rheumatology 49(11):2010-2015

Borghi C, Palazzuoli A, Landolfo M, and Cosentino E. 2019. Hyperuricemia: a novel old disorder-relationship and potential mechanisms in heart failure. Heart Fail Rev

Chen L, Chen DQ, Wang M, Liu D, Chen H, Dou F, Vaziri ND, and Zhao YY. 2017. Role of RAS/Wnt/beta-catenin axis activation in the pathogenesis of podocyte injury and tubulo-interstitial nephropathy. Chem Biol Interact 273(56-72

Conesa A, Madrigal P, Tarazona S, Gomez-Cabrero D, Cervera A, McPherson A, Szczesniak MW, Gaffney DJ, Elo LL, Zhang X, and Mortazavi A. 2016. A survey of best practices for RNA-seq data analysis. Genome Biol $17(13$

Debelle FD, Nortier JL, De Prez EG, Garbar CH, Vienne AR, Salmon IJ, Deschodt-Lanckman MM, and 
393

394

395

396

397

398

399

400

401

402

403

404

405

406

407

408

409

410

411

412

413

414

415

416

417

418

419

420

421

422

423

424

425

426

427

428

429

430

431

432

433

434

435

436

437

438

439

440

441

442

443

444
Vanherweghem JL. 2002. Aristolochic acids induce chronic renal failure with interstitial fibrosis in saltdepleted rats. $J$ Am Soc Nephrol 13(2):431-436

Engel B, Just J, Bleckwenn M, and Weckbecker K. 2017. Treatment Options for Gout. Dtsch Arztebl Int 114(13):215-222

Feig DI. 2014. Serum uric acid and the risk of hypertension and chronic kidney disease. Curr Opin Rheumatol 26(2):176-185

Iwata H, Nishio S, Yokoyama M, Matsumoto A, and Takeuchi M. 1989. Solubility of uric acid and supersaturation of monosodium urate: why is uric acid so highly soluble in urine? J Urol 142(4):1095-1098

Keebaugh AC, and Thomas JW. 2010. The evolutionary fate of the genes encoding the purine catabolic enzymes in hominoids, birds, and reptiles. Mol Biol Evol 27(6):1359-1369

Li J, Hong S, Chen W, Zuo E, and Yang H. 2019. Advances in detecting and reducing off-target effects generated by CRISPR-mediated genome editing. $J$ Genet Genomics

Lin Y, Zhu J, Wang Y, Li Q, and Lin S. 2017. Identification of differentially expressed genes through RNA sequencing in goats (Capra hircus) at different postnatal stages. PLoS One 12(8):e0182602

Liu D, Yun Y, Yang D, Hu X, Dong X, Zhang N, Zhang L, Yin H, and Duan W. 2019. What Is the Biological Function of Uric Acid? An Antioxidant for Neural Protection or a Biomarker for Cell Death. Dis Markers 2019(4081962

Lu J, Hou X, Yuan X, Cui L, Liu Z, Li X, Ma L, Cheng X, Xin Y, Wang C, Zhang K, Wang X, Ren W, Sun R, Jia Z, Tian Z, Mi QS, and Li C. 2018. Knockout of the urate oxidase gene provides a stable mouse model of hyperuricemia associated with metabolic disorders. Kidney Int 93(1):69-80

Ma L, Hu J, Li J, Yang Y, Zhang L, Zou L, Gao R, Peng C, Wang Y, Luo T, Xiang X, Qing H, Xiao X, Wu C, Wang Z, He JC, Li Q, and Yang S. 2018. Bisphenol A promotes hyperuricemia via activating xanthine oxidase. FASEB J 32(2):1007-1016

Mangoff SC, and Milner JA. 1978. Oxonate-induced hyperuricemia and orotic aciduria in mice. Proc Soc Exp Biol Med 157(1):110-115

Nakamura S, Watanabe S, Ando N, Ishihara M, and Sato M. 2019. Transplacental Gene Delivery (TPGD) as a Noninvasive Tool for Fetal Gene Manipulation in Mice. Int J Mol Sci 20(23)

Ramazzina I, Folli C, Secchi A, Berni R, and Percudani R. 2006. Completing the uric acid degradation pathway through phylogenetic comparison of whole genomes. Nat Chem Biol 2(3):144-148

Sapranauskas R, Gasiunas G, Fremaux C, Barrangou R, Horvath P, and Siksnys V. 2011. The Streptococcus thermophilus CRISPR/Cas system provides immunity in Escherichia coli. Nucleic Acids Res 39(21):92759282

Schlesinger N. 2017. The safety of treatment options available for gout. Expert Opin Drug Saf 16(4):429-436

Sharma V, and Hiller M. 2019. Losses of human disease-associated genes in placental mammals. NAR Genomics and Bioinformatics 2(1)

Shen W, Le S, Li Y, and Hu F. 2016. SeqKit: A Cross-Platform and Ultrafast Toolkit for FASTA/Q File Manipulation. PLoS One 11(10): 0163962

Stavric B, and Nera E. 1978. Use of the uricase-inhibited rat as an animal model in toxicology. Clin Toxicol 13(1):47-74

Trapnell C, Williams BA, Pertea G, Mortazavi A, Kwan G, van Baren MJ, Salzberg SL, Wold BJ, and Pachter L. 2010. Transcript assembly and quantification by RNA-Seq reveals unannotated transcripts and isoform switching during cell differentiation. Nat Biotechnol 28(5):511-515

Wu X, Wakamiya M, Vaishnav S, Geske R, Montgomery C, Jr., Jones P, Bradley A, and Caskey CT. 1994. Hyperuricemia and urate nephropathy in urate oxidase-deficient mice. Proc Natl Acad Sci U S A 91(2):742746

Yin H, Hou X, Tao T, Lv X, Zhang L, and Duan W. 2015. Neurite outgrowth resistance to rho kinase inhibitors in PC12 Adh cell. Cell Biol Int 39(5):563-576

Yun Y, Gao T, Li Y, Gao Z, Duan J, Yin H, and Duan W. 2016. Excretory Function of Intestinal Tract Enhanced in Kidney Impaired Rats Caused by Adenine. ScientificWorldJournal 2016(2695718

Yun Y, Yin H, Gao Z, Li Y, Gao T, Duan J, Yang R, Dong X, Zhang L, and Duan W. 2017. Intestinal tract is an important organ for lowering serum uric acid in rats. PLoS One 12(12):e0190194 
Figure 1

Uricase activity in serum (A) and liver homogenate (B).

Apart from A292 transiently increasing at the beginning of the reaction, A292 decreased in the reaction system containing serum uricase of wild-type rats. However, A292 continuously increased in the reaction system containing Uox ${ }^{-/}$rat serum. The pattern of uricase activity in liver homogenate of Uox ${ }^{-1 /}$ rats was similar to that in serum.
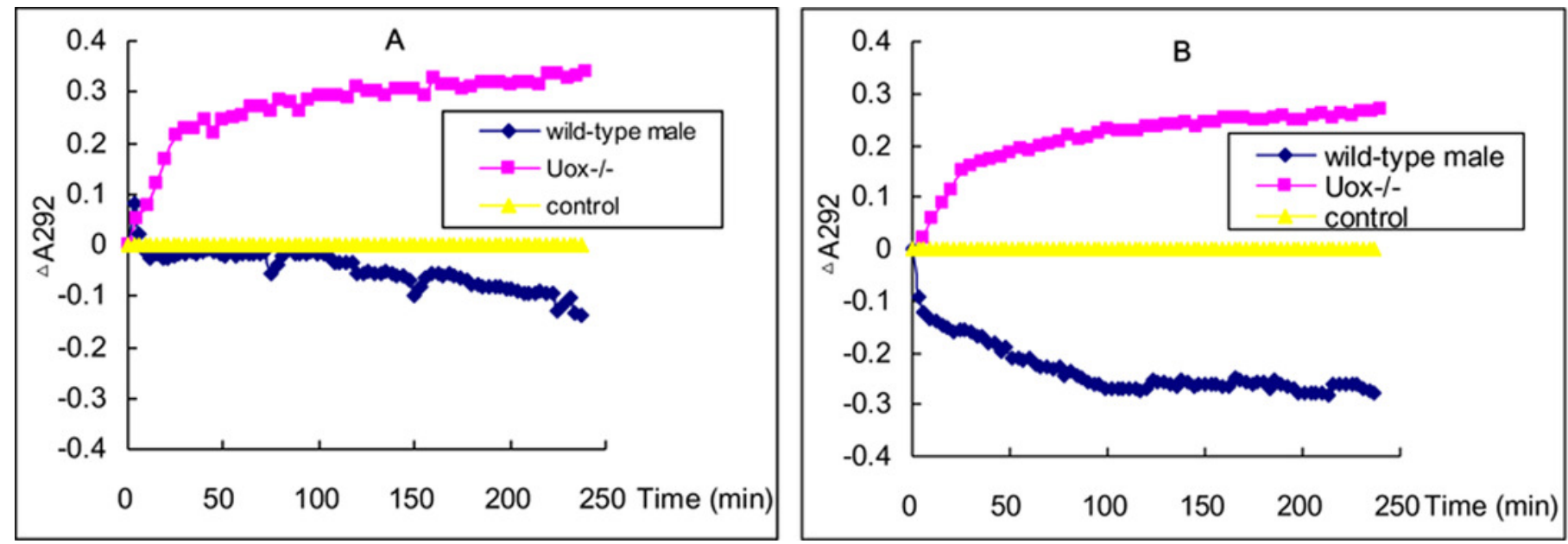


\section{Figure 2}

Uricase-deficient rat identified by Western blot (A) and RT-PCR (B).

Uricase protein from wild-type (WT) rat is about $35 \mathrm{kDa}$ and the encoded mRNA results in a PCR product of about $500 \mathrm{bp}$. As controls, $\beta$-actin (42 kDa) was both positive in Uox ${ }^{-/ .}$and WT rats, and so was GAPDH (glyceraldehyde 3 phosphate dehydrogenase) (149 bp) in RT-PCR. However, Uox ${ }^{-/}$rats express no normal uricase both at protein level and mRNA level.

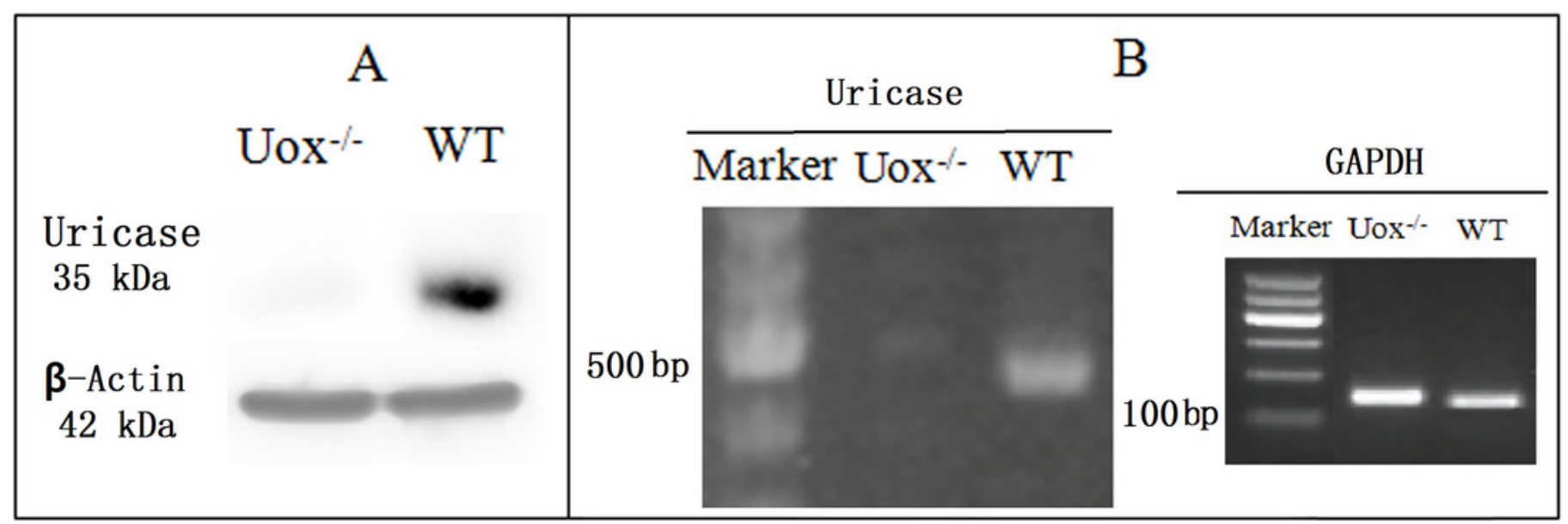




\section{Figure 3}

The alignment of RNA-seq reads to the rat Uox mRNA sequence from wild-type (WT) rat (A) and Uox ${ }^{-/-}$rat (B).

The horizontal axis was the reads, and vertical axis showed the sequence of the template mRNA (NM_053768.2). A striped bar means a fragment mapped by a read or the whole read. If there are two or more reads that mapped the same fragment, two or more striped bars were showed behind. The reads derived from wild-type rat almost fully covered the template mRNA (A). However, the nucleotide segment from Exon 2, 3, and 4 (71-477 nt) was missing in Uox ${ }^{-1-}$ rat $(B)$.
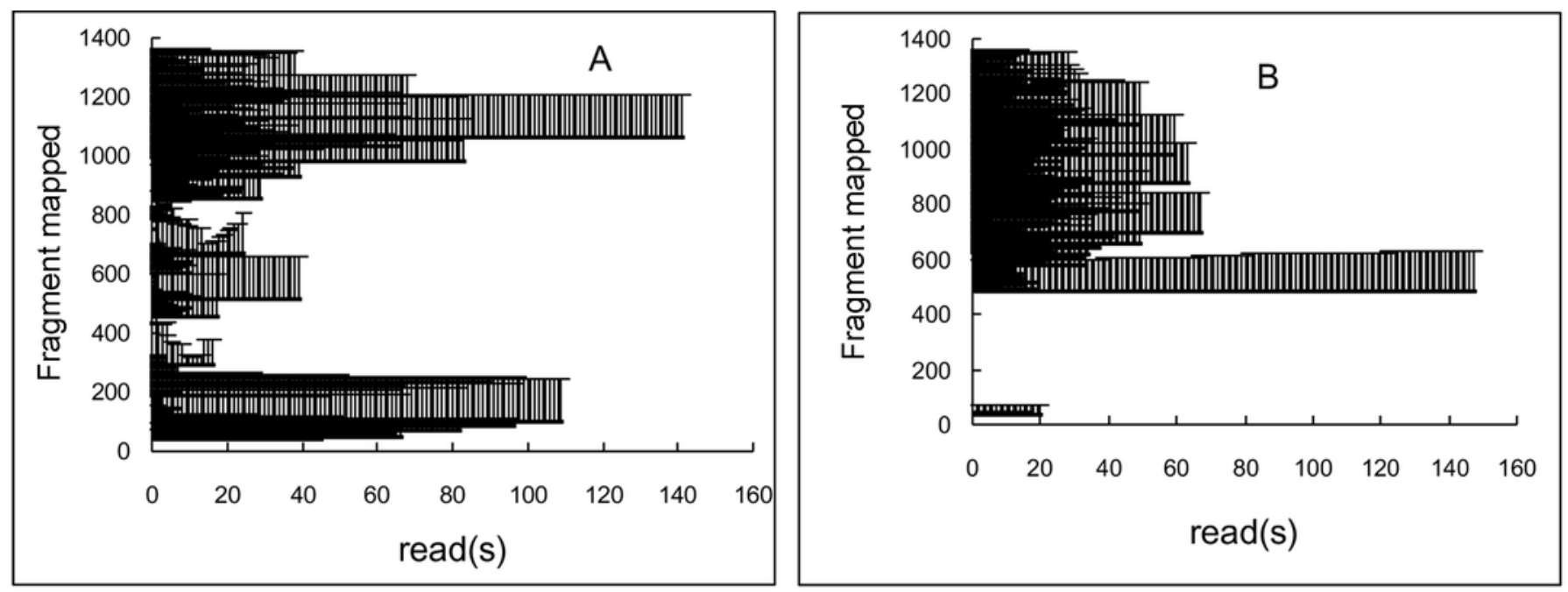
Figure 4

Biological features of Uox ${ }^{-1 /}$ rats 45 days after birth (Mean \pm SD).

A, Body weight of stable Uox ${ }^{-1-}$ rats (F3, F4, F5 and F6 animal) $[n=160$ (male), or 185

(female)]. B, Serum uric acid (SUA) in wild-type rats [ $=10$ (male), or 9 (female)]. C, SUA in adult Uox ${ }^{-1-}$ rats (F3, F4, F5 and F6 animal) [ $n=160$ (male), or 185 (female)]. D, Blood fat in wild type rats [ $n=20$ (10 male, 10 female) $]$ and in Uox ${ }^{-/}$rats $[n=12$ (7 male, 5 female) $] . P$ $<0.05$, independent Student's t-test (two tailed). TG, total triglyceride; TC, total cholesterol; $\mathrm{HDL}$, high density lipoprotein; LDL, low density lipoprotein.
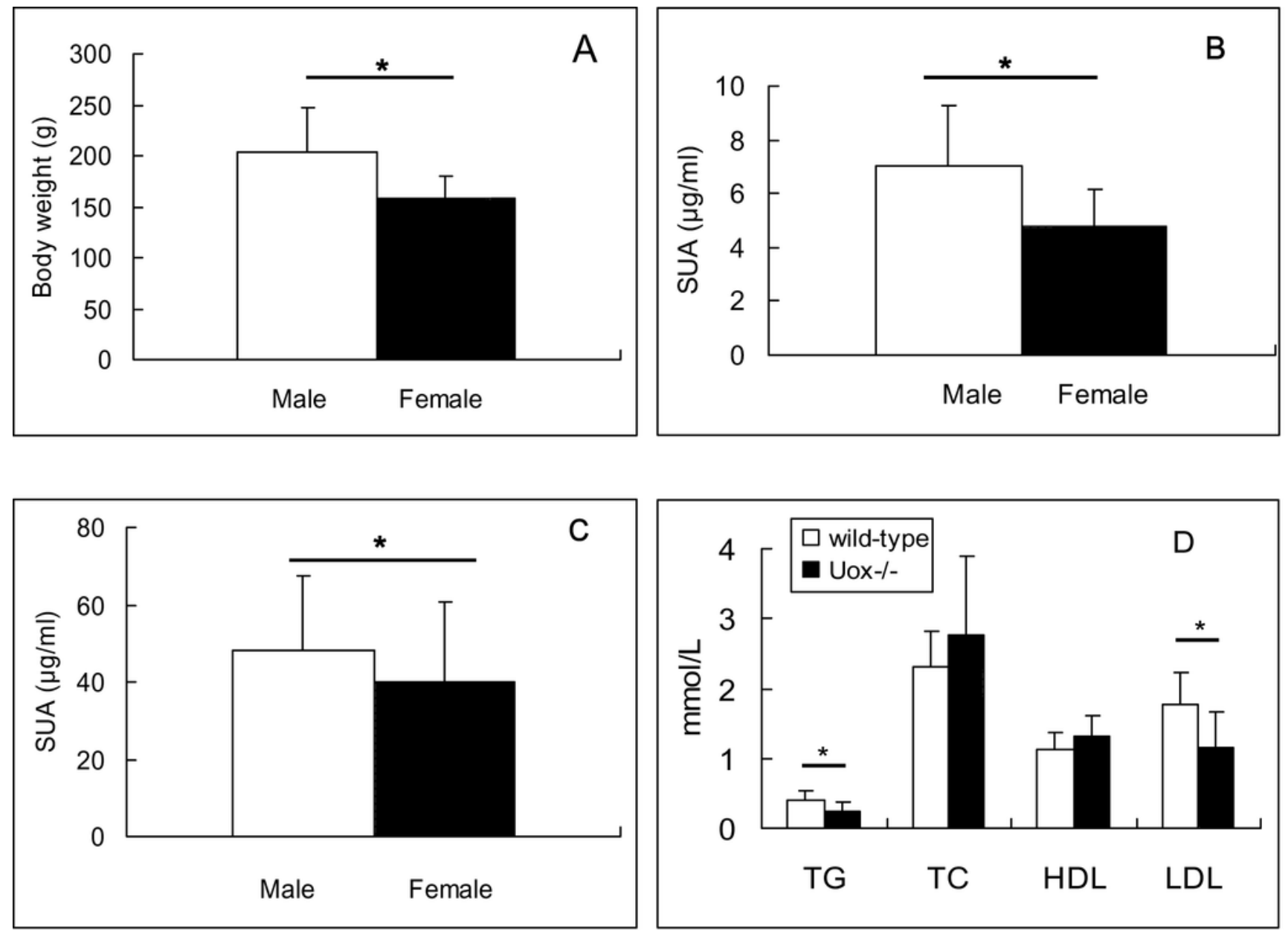


\section{Figure 5}

Kidney of male Uox ${ }^{-/}$rats and its function (Mean $\pm S D$ ).

Left kidney index (LKI) in Uox rats $(n=7)$ was bigger that that in wild-type rats $(n=10)(A)$. BUN in Uox rats $(n=10)$ was higher than that in wild-type rats $(n=5)(B)$. However, $\mathrm{Cr}$ in Uox ${ }^{-1}$ rats $(n=10)$ was lower than that in wild-type rats $(n=5)(C)$. Uric acid in Uox ${ }^{-1}$ rats' kidney $(n=9)$ was higher than that in male wild-type rats' $(n=8)(D)$. BUN, blood urea nitrogen; $\mathrm{Cr}$, serum creatinine. $* \mathrm{P}<0.05$, independent Student's t-test (two tailed)
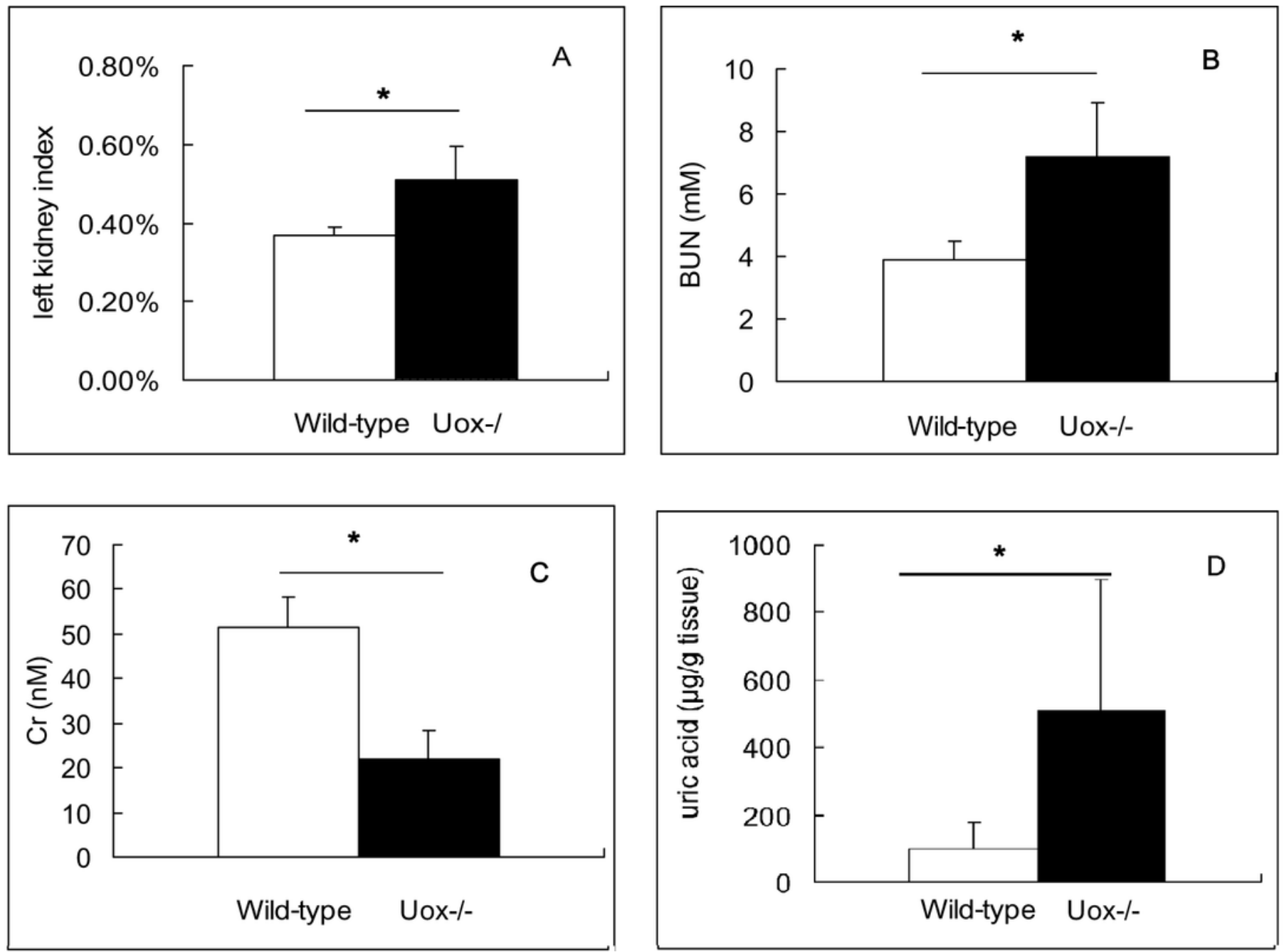


\section{Figure 6}

Microscopic histology of rat kidneys in male wild-type rats $(A, B)$ and Uox ${ }^{-1 /}$ rats $(C, D)$.

Nephrocytes in wild-type rats were piled tightly (A, B); and there were no swelling signs in glomeruli (A) and tubules (B). Meanwhile in Uox rats, cells in the glomeruli were slightly swollen and their capsular spaces were slightly dilated (C), the tubular walls became thin, and their spaces were also slightly dilated (D). Sporadic interstitial fibrosis and inflammatory cell infiltration can also be seen in the kidneys of Uox rats (C, D). Bar $=50 \mu \mathrm{m}$
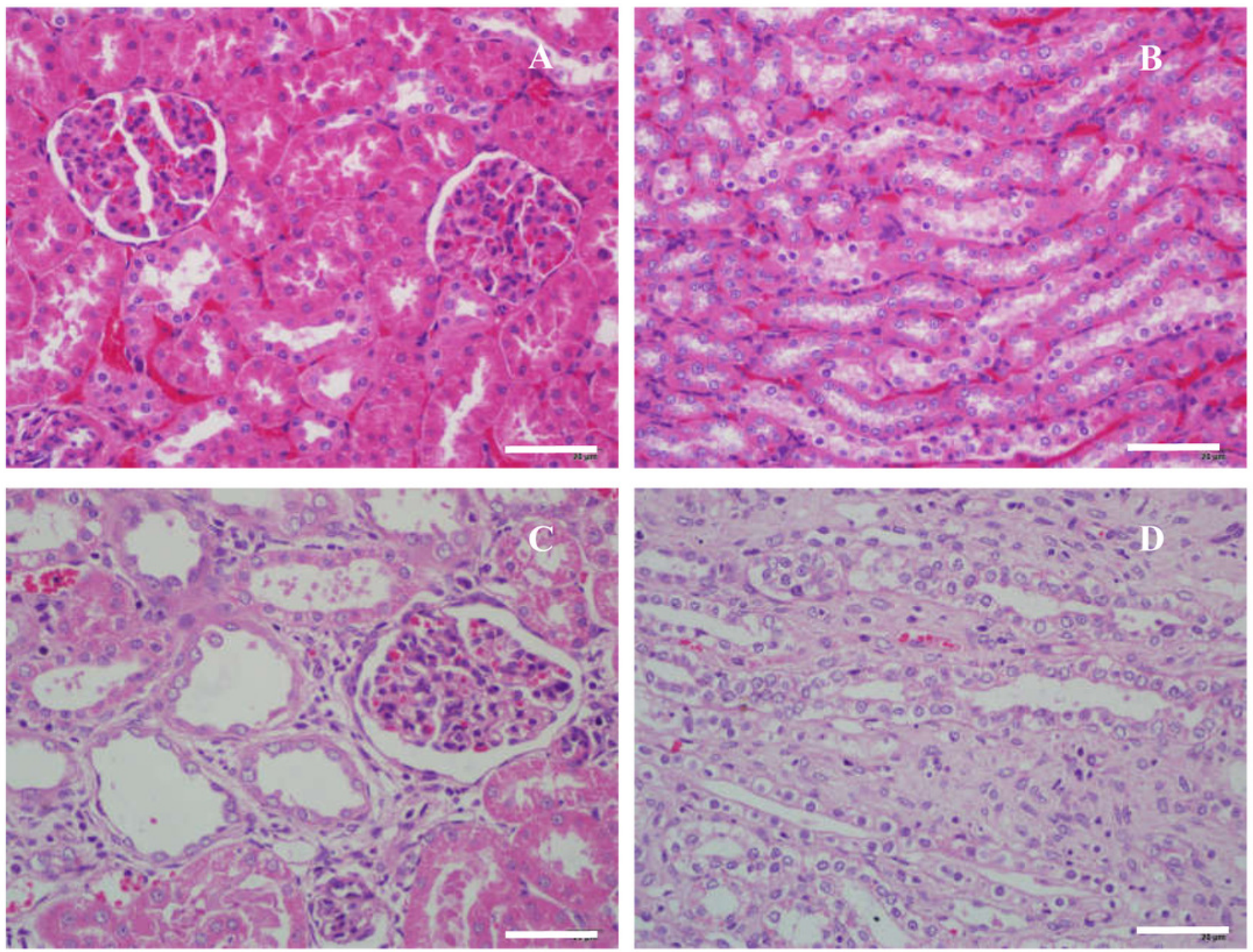


\section{Table $\mathbf{1}$ (on next page)}

SUA $(\mu \mathrm{g} / \mathrm{ml})$ of adult Uox ${ }^{+-}$rat. 


\begin{tabular}{ccccc}
\hline No. & gender & Serum uric acid (SUA) & Mean \pm SD & note \\
\hline 1 & Male & 20.91 & & Third round \\
2 & Male & 9.92 & $13.80 \pm 6.17$ & Third round \\
3 & Male & 10.57 & & Fourth round \\
\hline 4 & Female & 13.78 & $17.03 \pm 2.84$ & Fourth round \\
5 & Female & 19.00 & & Fourth round \\
\hline
\end{tabular}

1 


\section{Table 2 (on next page)}

Likely Uox ${ }^{-1-}$ rats screened out from F0 offspring*

a Male rats with SUA above $40 \mu \mathrm{g} / \mathrm{ml}$ or female rats with SUA above $30 \mu \mathrm{g} / \mathrm{ml}$ were recognized as likely Uox ${ }^{-/}$rats. b Likely Uox ${ }^{-/-}$rats died several days after birth. The dead rats were identified based on the uric acid in their kidney above $1000 \mu \mathrm{g} /(\mathrm{g}$ tissue). * The number of offspring (F1) was 6 (3/3, male/female), the number of F2 animal was 48 (22/26), the number of F3 animal was 105 [47/(58-1)], the number of F4 animal was 47 [(14-1)/33], the number of F5 animal was 70 (36/34), and the number of F6 animal was 75 (41/34). 


\begin{tabular}{ccccc}
\hline Cage & $\begin{array}{c}\text { Offspring } \\
(\text { Male/Female })\end{array}$ & $\begin{array}{c}\text { a, Likely Uox }{ }^{-/} \text {rats } \\
(\text { Male/Female })\end{array}$ & $\begin{array}{c}\text { other rats } \\
(\text { Male/Female })\end{array}$ & Note \\
\hline 1 & $9(4 / 5)$ & $7(2 / 5)$, died $^{\mathrm{b}}$ & $2(2 / 0)$ & From third round Uox $^{+/-}$rats \\
2 & $3(2 / 1)$ & $3(2 / 1)$, died $^{\mathrm{b}}$ & 0 & From third Uox $^{+/-}$rats \\
3 & $14(8 / 6)$ & $6(3 / 3)$ & $8(5 / 3)$ & ${\text { From fourth } \text { Uox }^{+/-} \text {rats }}$ \\
\hline
\end{tabular}

1 


\section{Table 3(on next page)}

Genes associated with uric acid transport or metabolism expressed in male rat kidneys (mean $\pm \mathrm{SD}, \mathrm{n}=3$ ).

FPKM, Fragment Per Kilobases of exon model per Million mapped reads. $* \mathrm{P}<0.05$, independent Student's t-test, two-tailed. ${ }^{*}$ the expression of Uox in Uox ${ }^{-1}$ rat is invalidated. 


\begin{tabular}{|c|c|c|c|c|c|c|c|}
\hline \multirow{2}{*}{ No } & \multirow{2}{*}{ Gene } & \multicolumn{2}{|c|}{ FPKM } & \multirow{2}{*}{$\begin{array}{c}P \\
\text { (a vs b) }\end{array}$} & \multirow{2}{*}{ Fold(b/a) } & \multirow{2}{*}{ Alias } & \multirow{2}{*}{ Note } \\
\hline & & a wild-type & b Uox ${ }^{-1-}$ & & & & \\
\hline 1 & Abcg2 & $71.19 \pm 10.03$ & $79.90 \pm 8.91$ & 0.324 & 1.12 & BCRP1 & secretion \\
\hline 2 & Abcc 4 & $17.17 \pm 2.02$ & $13.89 \pm 1.17$ & 0.072 & 0.81 & MRP4 & secretion \\
\hline 3 & Lgals9 & $13.62 \pm 2.34$ & $7.40 \pm 1.77$ & $0.021^{*}$ & 0.54 & UAT & secretion \\
\hline 4 & Slc17a1 & $44.01 \pm 4.75$ & $54.49 \pm 6.00$ & 0.077 & 1.24 & NPT1 & secretion \\
\hline 5 & Slc22a6 & $541.93 \pm 36.04$ & $505.83 \pm 51.18$ & 0.374 & 0.93 & OA1 & secretion \\
\hline 6 & Slc2a9 & $7.21 \pm 0.36$ & $7.62 \pm 0.37$ & 0.237 & 1.06 & GLUT9 & reclamation \\
\hline 7 & Slc2a6 & $1.03 \pm 0.057$ & $0.88 \pm 0.094$ & 0.076 & 0.85 & GLUT9 & reclamation \\
\hline 8 & Slc22a13 & $11.67 \pm 3.25$ & $3.42 \pm 0.05$ & $0.012^{*}$ & 0.29 & OAT10 & reclamation \\
\hline 9 & Slc22a8 & $395.78 \pm 47.31$ & $433.38 \pm 31.86$ & 0.317 & 1.10 & OAT3 & reclamation \\
\hline 10 & Slc22a12 & $396.99 \pm 33.77$ & $285.08 \pm 43.38$ & $0.024 *$ & 0.72 & URAT1 & reclamation \\
\hline 11 & $\mathrm{Xdh}$ & $35.45 \pm 3.79$ & $37.95 \pm 3.84$ & 0.467 & 1.07 & UOR & synthesis \\
\hline 12 & Uox $^{\#}$ & $0.03 \pm 0.06$ & $0.01 \pm 0.01$ & 0.507 & 0.25 & UOX & degradation \\
\hline 13 & Ada & $9.59 \pm 2.00$ & $8.01 \pm 1.62$ & 0.347 & 0.84 & $\mathrm{ADA}$ & synthesis \\
\hline
\end{tabular}

1 
Table 4 (on next page)

Scores of renal microscopic pathology $(n=3)$.

*Mann-Whitney U model (two tailed) 


\begin{tabular}{lcccccc}
\hline \multirow{2}{*}{ Item } & \multicolumn{3}{c}{ Wild-type } & \multicolumn{4}{c}{ Uox $^{-/-}$} \\
\cline { 2 - 7 } & Sample1 & Sample2 & Sample3 & Sample1 & Sample2 & Sample3 \\
\hline Glomerular abnormality & 0 & 0 & 0 & 1 & 1 & 1 \\
Tubular atrophy & 0 & 0 & 0 & 1 & 1 & 1 \\
Tubular necrosis & 0 & 0 & 0 & 1 & 1 & 1 \\
Lymphocytic infiltrates & 0 & 0 & 0 & 1 & 1 & 1 \\
Interstitial fibrosis & 0 & 1 & 0 & 1 & 1 & 1 \\
\hline sum & 0 & 1 & 0 & 5 & 5 & 5 \\
\hline $\mathrm{P}^{*}$ & & & & 0.034 & & \\
\hline
\end{tabular}

1 